\title{
VALIDACIÓN DE UN MÉTODO ANALÍTICO PARA CUANTIFICAR ALUMINIO EN TEJIDOS DE RATAS SPRAGUE DAWLEY MEDIANTE EAA-FLAMA Y EAA-HORNO DE GRAFITO
}

\author{
Alejandro Monserrat GARCÍA-ALEGRÍA ${ }^{1}$, Humberto Francisco ASTIAZARÁN-GARCÍA ${ }^{2}$, \\ Iván ANDURO-CORONA ${ }^{2}$, Armando BURGOS-HERNÁNDEZ ${ }^{3}$, Eduardo RUIZ-BUSTOS ${ }^{4}$, \\ Rafael CANETT-ROMERO ${ }^{3}$, Kareen Krizzan ENCINAS-SOTO 5 y Agustín GÓMEZ-ÁLVAREZ ${ }^{5 *}$
}

\footnotetext{
${ }^{1}$ Programa de Doctorado en Ciencias (Químico Biológicas y de la Salud), Universidad de Sonora, Luis Encinas y Rosales s/n, Colonia Centro, 83000 Hermosillo, Sonora, México

${ }^{2}$ Dirección de Nutrición, Centro de Investigación en Alimentación y Desarrollo, A.C., km 0.6 carretera a La Victoria, 83304 Hermosillo, Sonora, México

${ }^{3}$ Departamento de Investigación y Posgrado en Alimentos, Universidad de Sonora, Luis Encinas y Rosales s/n, Colonia Centro, 83000 Hermosillo, Sonora, México

${ }^{4}$ Departamento de Ciencias Químico Biológicas, Universidad de Sonora, Luis Encinas y Rosales s/n, Colonia Centro, 83000 Hermosillo, Sonora, México

${ }^{5}$ Departamento de Ingeniería Química y Metalurgia, Universidad de Sonora, Luis Encinas y Rosales s/n, Colonia Centro, 83000 Hermosillo, Sonora, México

*Autor para correspondencia: agustin.gomez@unison.mx
}

(Recibido agosto 2017; aceptado diciembre 2017)

Palabras clave: espectroscopia de absorción atómica, metales, roedores

\section{RESUMEN}

Se validó el método para cuantificar aluminio $(\mathrm{Al})$ en tejidos de ratas Sprague Dawley mediante espectroscopia de absorción atómica por flama (EAA-F) y por horno de grafito (EAA-HG). La linealidad se evaluó mediante el coeficiente de correlación $(r)$, cuyos resultados fueron de 1.000 y 0.9993 para EAA-F y EAA-HG, respectivamente. La exactitud fue de 100.62 y $97.75 \%$ de recuperación, en tanto que la precisión fue de 0.61 y $1.30 \%$ del coeficiente de variación para EAA-F y EAA-HG, respectivamente. La precisión también se evaluó en condiciones de repetibilidad; el resultado obtenido indica que el método es repetible para las concentraciones de aluminio $(\mathrm{Al})$ utilizadas y determinadas por EAA-F y EAA-HG. Los resultados de límite de detección y límite de cuantificación para Al determinados por EAA-F fueron de 0.06 y $0.19 \mathrm{mg} / \mathrm{L}$, y de 0.05 y $0.18 \mu \mathrm{g} / \mathrm{L}$ por EAA-HG. Por otro lado, se evaluó la exactitud del método analítico con un material de referencia certificado de tejido de ostión (NIST-1566b) digerido en horno de microondas, el cual contiene una concentración $197.2 \pm 6.0 \mathrm{mg}$ de $\mathrm{Al} / \mathrm{Kg}$ de tejido. Los resultados obtenidos para la exactitud a través del porcentaje de recuperación fueron de $100.60 \%$ para EAA-F y de $100.51 \%$ para EAA-HG. La aplicabilidad del método se evaluó mediante la fortificación de tejidos de ratas (mama, cerebro, hígado y hueso), así como controles digeridos en horno de microondas. Los resultados obtenidos para la exactitud mediante el porcentaje de recuperación fluctuaron entre 99.32 y 109.00 . 
Key words: atomic absorption spectroscopy, metals, rodents

\begin{abstract}
A method to quantify aluminum (Al) in tissues of Sprague Dawley rats by flame atomic absorption spectrophotometry (F-AAS) and graphite furnace (GF-AAS) was validated. The linearity was evaluated by the correlation coefficient $(\mathrm{r})$ and the results were 1.000 and 0.9993 for F-AAS and GF-AAS, respectively. Accuracy was 100.62 and $97.75 \%$, while the precision coefficient of variation was of 0.61 and $1.30 \%$ for F-AAS and GF-AAS, respectively. Precision was also evaluated under repeatability conditions; the result obtained indicates that the method is repeatable for the Al concentrations used and determined by F-AAS and GF-AAS. The results of limit of detection and limit of quantification of Al determined by F-AAS were 0.06 and $0.19 \mathrm{mg} / \mathrm{L}$, and 0.05 to $0.18 \mu \mathrm{g} / \mathrm{L}$ of Al determined by GF-AAS. Furthermore, the accuracy of the analytical method was evaluated with oyster tissue as certified reference material (NIST-1566b) digested in microwave system and containing a concentration of $197.2 \pm 6.0 \mathrm{mg}$ of Al$/ \mathrm{kg}$ of tissue. The results for accuracy through the percent recovery rate were 100.60 and $100.51 \%$ for F-AAS and GF-AAS, respectively. Finally, the applicability of the method was evaluated by fortifying rats' tissues and controls. The results for accuracy by the recovery rate ranged between 99.32 and $109.00 \%$.
\end{abstract}

\section{INTRODUCCIÓN}

El aluminio (Al) es un elemento muy abundante en la naturaleza y tiene una gran diversidad de usos industriales, domésticos y de consumo humano (Krewsky et al. 2007). Recientemente se ha propuesto el término "metales tóxicos" sobre la base de la alta solubilidad de estos elementos en agua y sus efectos adversos sobre la salud humana (Tchounwou et al. 2012).

Estimaciones recientes realizadas en EUA establecen que una persona adulta joven y de clase media consume alimentos y bebidas que contienen en promedio de 105 a $150 \mathrm{mg}$ de Al/día. Esto significa un consumo frecuente de este metal en sus diferentes formas y/o presentaciones de manera rutinaria (ATSDR 2008).

La incidencia de este factor ambiental sobre la población ha hecho que este metal sea objeto de múltiples investigaciones debido a su potencial toxicidad en la salud humana (ATSDR 2008). Algunos investigadores a nivel mundial han enfocado sus proyectos de investigación a demostrar la posible participación del Al como agente genotóxico que puede provocar inestabilidad genómica (di Virgilio et al. 2010). La inestabilidad genómica a su vez se puede relacionar directamente con carcinogénesis, además de toxicidad neurológica, hepática, renal y hematopoyética, entre otras (Prozialeck et al. 2008).
Debido en parte a que la gran mayoría de los estudios relacionados con los daños potenciales del $\mathrm{Al}$ se han basado en el uso de animales de experimentación, como ratas y ratones (Yamamoto et al. 2007, Yokel et al. 2008), las metodologías analíticas utilizadas para la cuantificación de este metal en tejidos animales son variadas.

Entre éstas destaca la espectroscopia de absorción atómica (EAA) (Mahieu et al. 2009), que no ha sido validada con anterioridad para diferentes tejidos en ratas, por lo que no existe plena confianza en los resultados obtenidos al cuantificar aluminio en dichos tejidos, de acuerdo con lo establecido en la normatividad internacional (ISO 2005). Esto permite tener la certeza de la comprobación de los resultados con relación a la evaluación de materiales de referencia certificados que contienen $\mathrm{Al}$ en tejidos biológicos.

Por tal razón, el principal objetivo de este trabajo fue validar el procedimiento analítico para cuantificar $\mathrm{Al}$ en tejidos de ratas Sprague Dawley mediante EAA por flama (EAA-F) y por horno de grafito (EAA-HG). Esto con la finalidad de disponer de un método analítico confiable sobre la base del proceso de validación, que pueda servir como método de referencia para cuantificar $\mathrm{Al}$ en tejidos de ratas y en otros animales de experimentación.

Una vez realizada la validación del método, se podrá estimar la incertidumbre expandida, lo cual 
generará mayor confiabilidad respecto del resultado obtenido durante la determinación de un mensurado, como es el caso de $\mathrm{Al}$ en tejidos de ratas.

\section{MATERIALES Y MÉTODOS}

\section{Equipo}

Se utilizó un equipo de EAA Perkin Elmer modelo AAnalyst 400, equipado con un horno de grafito Perkin Elmer modelo HG 900 con automuestreador AS 800. Se utilizó lámpara mono-elemento de aluminio, sistema de corrección de fondo y tubos de grafito integrados con plataforma Lvov (Perkin Elmer 2012).

Adicionalmente, se cuantificó Al por EAA por flama de óxido nitroso-acetileno. Las condiciones de operación se indican en el cuadro I. Para la descomposición de las muestras de tejidos de animales, se utilizó un equipo de horno de microondas MARSxpress 5 CEM Corporation (Mathews, NC, EUA).

CUADRO I. CONDICIONESDEOPERACIÓNDELEQUIPO DE ESPECTROSCOPIADEABSORCIÓNATÓMICAPERKIN ELMER MODELOAANALYST 400

\begin{tabular}{lc}
\hline Horno de grafito modelo HG 900 & Parámetros \\
\hline Longitud de onda $(\lambda)$ & $309.3 \mathrm{~nm}$ \\
Gas de purga & Argón \\
Rampas: & $150^{\circ} \mathrm{C}$ \\
$\quad$ Secado & $1700^{\circ} \mathrm{C}$ \\
Pirolisis & $2400^{\circ} \mathrm{C}$ \\
Atomización & $2600^{\circ} \mathrm{C}$ \\
Limpieza & $\mathrm{Mg}\left(\mathrm{NO}_{3}\right)_{2} 0.2 \%$ \\
Eliminación de interferencias & $\mathrm{Std} \mathrm{Al} 20 \mu \mathrm{g} / \mathrm{L}=0.18$ \\
Sensitividad & absorbancia \\
& Parámetros \\
\hline Flama & $309.3 \mathrm{~nm}$ \\
\hline Longitud de onda $(\lambda)$ & Cátodo hueco de $\mathrm{Al}$ \\
Lámpara & Óxido nitroso/acetileno \\
Oxidante-comburente & $0.7 \mathrm{~mm}$ \\
Apertura & $\mathrm{KCl} 0.2 \%$ \\
Eliminación de interferencias & Estándar de $\mathrm{Al} 50 \mathrm{mg} / \mathrm{L}=$ \\
Sensibilidad & 0.22 absorbancia \\
\hline
\end{tabular}

Fuente: Perkin Elmer (2014)

\section{Calibración del equipo de EAA}

En la cuantificación de aluminio en soluciones estándares, material de referencia certificado, muestras de tejido de ratas, agua desionizada y alimento, se utilizó un equipo EAA Perkin-Elmer Modelo
AAnalyst 400, tanto para flama como para horno de grafito. La calibración se realizó mediante la prueba de sensibilidad como se indica en el cuadro I.

\section{Reactivos, estándares y material de referencia certificado}

Los reactivos $\mathrm{Mg}\left(\mathrm{NO}_{3}\right)_{2}, \mathrm{HNO}_{3}$ concentrado y $\mathrm{KCl}$ fueron obtenidos de J.T. Baker (Center Valley, PA, EUA). Se utilizó un estándar de aluminio de 1000 $\pm 2 \mathrm{mg} / \mathrm{L}$ (AccuStandard, New Haven, CT, EUA), un material de referencia certificado (CRM-S-B) que contiene $700 \pm 4 \mathrm{mg} / \mathrm{L}$ de Al (High-Purity Standard, Charleston, SC, EUA) y un material de referencia certificado de tejido de ostión (del National Institute of Standards and Technology estadounidense [NIST1566b, Gaithersburg, MD, US]) que contiene 197.2 $\pm 6 \mathrm{mg} / \mathrm{kg}$ de $\mathrm{Al}$.

\section{Preparación de materiales de cristalería}

El material fue sometido a un estricto control para evitar la contaminación por elementos traza, siguiendo la metodología recomendada por van Loon (1985) y Bohrer et al. (2008).

Los materiales de trabajo de vidrio (pipetas serológicas, volumétricas y matraces volumétricos, entre otros) se lavaron exhaustivamente con detergente líquido comercial para después ser sometidos en una solución de $\mathrm{HNO}_{3}$ al $20 \%$ (J.T. Baker) durante tres días. Posteriormente fueron enjuagados con agua desionizada. Una vez secado, el material se guardó en recipientes de plástico con tapa hermética hasta su uso.

\section{Calibración de balanza analítica, material volu- métrico y micropipetas}

El material volumétrico, así como las micropipetas (High Tech Lab) fueron calibrados mediante el método gravimétrico (Batista et al. 2009, Lorefice 2009, Liang et al. 2013), con una balanza analítica marca Vibra modelo AF-R220E 220/0.0001g $(\mathrm{Max} / \mathrm{d})$. Esta balanza previamente fue calibrada con un juego de masas patrón de laboratorio $(0.2,0.5,1$ y $100 \mathrm{~g}$ ) marca Rice Like, certificadas por la Entidad Mexicana de Acreditación (EMA).

\section{Validación del método}

Los parámetros analíticos de calidad que se han considerado para la validación del método son los siguientes:

Linealidad. Se comprobó a través de curvas de calibración y estimación del coeficiente de correlación $(r)$ y de la pendiente $(m)$. Para ello, se prepararon tres curvas de calibración a partir de un estándar 
de $\mathrm{Al}$ de alta pureza (Accustandard) que contiene $1000 \mathrm{mg} / \mathrm{L}$ de Al.

Concentraciones de trabajo. Fueron de 10, 20, 50, 100 y $200 \mathrm{mg} / \mathrm{L}$ de $\mathrm{Al}$, las cuales se acondicionaron con $\mathrm{HNO}_{3}$ al $2 \%$ (J.T. Baker) y con $\mathrm{KCl}$ al $0.2 \%$ (J.T. Baker) para su cuantificación con EAA-F con óxido nitroso-acetileno. Adicionalmente se prepararon tres curvas de calibración a concentraciones de 20, 40, 60, 80 y $100 \mu \mathrm{g} / \mathrm{L}$ de $\mathrm{Al}$, y se acondicionaron con $\mathrm{HNO}_{3}$ al $2 \%$ y con $\mathrm{Mg}\left(\mathrm{NO}_{3}\right)_{2}$ al $0.2 \%$ (J.T. Baker) para su cuantificación por EAA-HG.

Exactitud y precisión. Estas variables se determinaron a través del porcentaje de recuperación $(\% \mathrm{Rec})$ y el porcentaje de coeficiente de variación $(\% \mathrm{CV})$, respectivamente. Para ello, se utilizó el material de referencia certificado CRM-S-B (sedimento de suelo en solución acuosa, High-Purity Standards, Charleston SC, EUA), que contiene $700 \pm 4 \mathrm{mg} / \mathrm{L}$ de $\mathrm{Al}$.

Posteriormente se preparó un estándar de trabajo de $70 \mathrm{mg} / \mathrm{L}$ de Al, el cual se acondicionó con $\mathrm{HNO}_{3}$ al $2 \%$ y con $\mathrm{KCl}$ al $0.2 \%$ para EAA-F. Para EAA-HG, el estándar de trabajo se preparó con una concentración de $70 \mu \mathrm{g} / \mathrm{L}$ de $\mathrm{Al}$ y se acondicionó con $\mathrm{HNO}_{3}$ al $2 \%$ y con $\mathrm{Mg}\left(\mathrm{NO}_{3}\right)_{2}$ al $0.2 \%$.

Repetibilidad. Se determinó mediante análisis de varianza, utilizando el estadístico de prueba $\mathrm{F}$ con un nivel de significancia del $5 \%(\alpha=0.05)$ y $n=25$. Para esto, se prepararon estándares de trabajo por quintuplicado por semana durante un tiempo total de cinco semanas a concentraciones de 20,50 y 100 $\mathrm{mg} / \mathrm{L}$ de $\mathrm{Al}$, los cuales se acondicionaron con $\mathrm{HNO}_{3}$ al $2 \%$ y $\mathrm{KCl}$ al $0.2 \%$ para EAA-F.

Para EAA-HG, se utilizaron estándares de trabajo a concentraciones por triplicado de 20, 40 y $60 \mu \mathrm{g} / \mathrm{L}$ de $\mathrm{Al}$, que se acondicionaron con $\mathrm{HNO}_{3}$ al $2 \%$ y $\mathrm{Mg}\left(\mathrm{NO}_{3}\right)_{2}$ al $0.2 \%$. El criterio de aceptación de la repetibilidad consiste en que $F_{\text {calculada }} \leq F_{\text {tablas }}$ (Blair y Taylor 2007).

Límite de detección (LD) y límite de cuantificación (LC). Se obtuvieron a partir de los datos de las curvas de calibración para EAA-F y para EAA-HG. El LD se calculó a partir de $3 \mathrm{~s} / \mathrm{m}$, donde $s$ es la desviación estándar de 10 mediciones del blanco y $m$ es la pendiente de la curva de calibración (ISO 1994, 2005).

El LC fue calculado como $10 \mathrm{~s} / \mathrm{m}$. El blanco para EAA-F fue una solución de $\mathrm{HNO}_{3}$ al $2 \%$ y con $\mathrm{KCl}$ $0.2 \%$, en tanto que el blanco para EAA-HG fue una solución de $\mathrm{HNO}_{3}$ al $2 \%$ y $\mathrm{Mg}\left(\mathrm{NO}_{3}\right)_{2}$ al $0.2 \%$ (ISO 2005, Blair y Taylor 2007).

El proceso de validación del método analítico se completó mediante la utilización de un material de referencia certificado de tejido de ostión
(NIST-1566b) que contiene $197.2 \pm 6.0 \mathrm{mg}$ de $\mathrm{Al} / \mathrm{kg}$ de tejido y que reemplaza al tejido de rata, ya que no existe actualmente este tipo de tejido como material de referencia certificado.

En esta etapa se evaluó la exactitud a través del porcentaje de recuperación $(\% \mathrm{Rec})$ y la precisión a través del porcentaje del coeficiente de variación $(\% \mathrm{CV})$.

\section{Aplicabilidad del método validado}

El método analítico validado se empleó para cuantificar la concentración de $\mathrm{Al}$ en tejidos de ratas sin fortificar y fortificados con $100 \mathrm{mg} / \mathrm{L}$ de $\mathrm{Al}$ para EAA-F y con $200 \mu \mathrm{g} / \mathrm{L}$ de Al para EAA-HG.

\section{Condiciones de bioterio}

Se seleccionó un grupo de 10 ratas hembra Sprague Dawley proporcionadas por el bioterio del Departamento de Investigación y Posgrado en Alimentos de la Universidad de Sonora.

Las ratas, de $200 \pm 20 \mathrm{~g}$ de peso corporal y $\sin$ algún tipo de tratamiento específico, fueron mantenidas en condiciones estándar de bioterio, con ciclos de luz/oscuridad de $12 \mathrm{~h}$, humedad de 40 a $70 \%$, temperatura de 18 a $22{ }^{\circ} \mathrm{C}$, agua y alimento de libre consumo (Bohrer et al. 2008). El alimento fue adquirido de Formulab Diet (5008C33, San Luis MO, EUA) y el agua estuvo libre de Al.

\section{Obtención de muestras biológicas}

Las ratas Sprague Dawley fueron anestesiadas con halotano y eutanizadas por dislocación cervical, apegados a lo que establece la normatividad internacional, para evitar el sufrimiento animal (EMEA 2009, FDA 2014). Los tejidos se obtuvieron mediante disección con bisturí de acero inoxidable.

Se realizó una mezcla de cada grupo de tejidos para su molienda y posteriormente proceder a su descomposición. Los materiales utilizados fueron previamente lavados y desmineralizados tal y como lo establece la normatividad vigente en México (CENAM-EMA 2012).

\section{Descomposición de las muestras de tejidos de ratas Sprague-Dawley fortificadas con aluminio, testigos y del material de referencia certificado (NIST 1566b)}

Los tejidos de rata, al igual que el alimento, el agua de beber y el material de referencia certificado (tejido de ostión NIST-1566b) se digirieron en un equipo de horno de microondas MARSxpress 5 (CEM Corporation, Mathews, NC, EUA) (CEM 2014). Para ello, se pesaron por triplicado en tubos 
de teflón de $20.0 \times 4.0 \mathrm{~cm}, 0.500 \pm 0.05 \mathrm{~g}$ de mama, hígado, hueso y cerebro, así como alimento, agua de beber y tejido de ostión como controles.

A estas muestras se les agregaron $10 \mathrm{~mL}$ de estándar de $\mathrm{Al}$ de $1000 \mathrm{mg} / \mathrm{L}$ (Accustandard) y se digirieron con $10 \mathrm{~mL}$ de $\mathrm{HNO}_{3}$ concentrado (J.T.Baker) en horno de microondas MARSxpress 5 con un programa de digestión de $200{ }^{\circ} \mathrm{C} / 35 \mathrm{bar} / 1600 \mathrm{~W} / 15$ min. El extracto ácido resultante se aforó a $100 \mathrm{~mL}$ con agua desionizada.

Para EAA por horno de grafito se pesaron 0.250 $\pm 0.025 \mathrm{~g}$ de los mismos tejidos de rata, así como alimento y agua de beber. A estas muestras se les agregó $1 \mathrm{~mL}$ de estándar de $\mathrm{Al}$ de $10 \mathrm{mg} / \mathrm{L}$ y se digirieron con $5 \mathrm{~mL}$ de $\mathrm{HNO}_{3}$ concentrado (J.T.Baker) en horno de microondas MARSxpress 5, con las mismas condiciones de digestión señaladas.

El residuo ácido resultante se aforó con $50 \mathrm{~mL}$ con agua desionizada. La concentración de $\mathrm{Al}$ se cuantificó mediante EAA-F y EAA-HG. Para flama se utilizó $\mathrm{KCl}$ al $0.2 \%$ para eliminar la interferencia de ionización, y para horno de grafito se utilizó $\mathrm{Mg}\left(\mathrm{NO}_{3}\right)_{2}$ al $0.2 \%$ como modificador químico. En esta etapa se evaluó la exactitud a través del porcentaje de recuperación $(\% \mathrm{Rec})$ y la precisión a través del porcentaje del coeficiente de variación $(\% \mathrm{CV})$.

Adicionalmente se prepararon tres réplicas de blancos de digestión con agua desionizada digeridas en horno de microondas en las mismas condiciones de operación que las muestras y los testigos, acondicionadas con $\mathrm{HNO}_{3}$ al $2 \%$ y $\mathrm{KCl}$ al $0.2 \%$ para EAA-F, en tanto que para EAA-HG se acondicionaron con $\mathrm{HNO}_{3}$ al $2 \%$ y $\mathrm{Mg}\left(\mathrm{NO}_{3}\right)_{2}$ al $0.2 \%$ (Perkin Elmer 2012).

\section{RESULTADOS Y DISCUSIÓN}

\section{Validación del método}

Linealidad. El proceso de validación del método analítico se valoró en primera instancia a partir de la linealidad, la cual se evaluó para EAA por flama y horno de grafito. En la figura 1 se puede apreciar que el coeficiente de correlación $(r)$ fue de 1.000, lo cual indica que la asociación entre concentración de Al y absorbancia es muy estrecha para las concentraciones de 10, 20, 50, 100 y $200 \mathrm{mg} / \mathrm{L}$, en la variante analítica de EAA-F.

En la figura 2 se muestra que la linealidad mediante coeficiente de correlación ( $r$ ) fue de 0.9993 para la variante analítica de EAA-HG. Ambas pendientes $(m)$ tienen el mismo valor de 0.004. Estos resultados indican que este parámetro analítico es

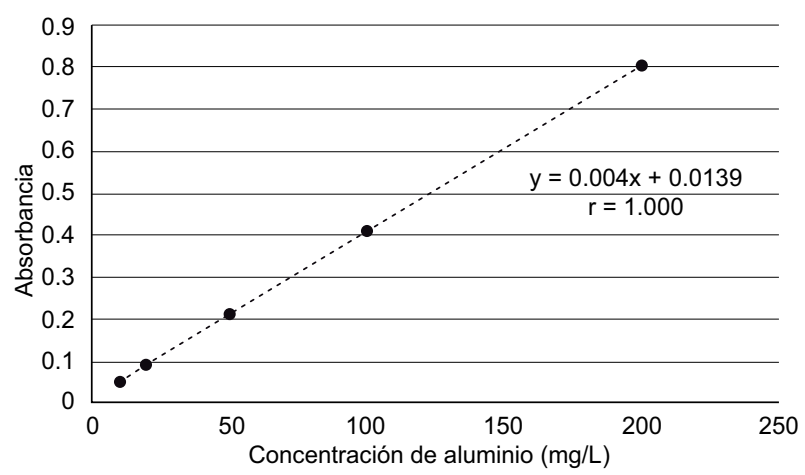

Fig. 1. Curva de calibración para Al cuantificado por espectroscopia de absorción atómica por flama $(\mathrm{mg} / \mathrm{L}), n=15$

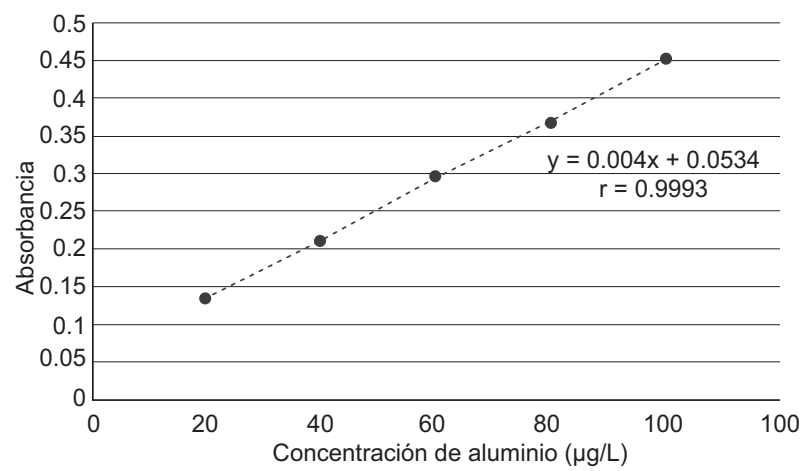

Fig. 2. Curva de calibración para Al cuantificado por espectroscopia de absorción atómica por horno de grafito $(\mu \mathrm{g} / \mathrm{L})$, $n=15$

aceptable, ya que el criterio de aceptación es de $r \geq$ 0.995 (Thompson et al. 2002).

Los resultados obtenidos para la linealidad son similares a los obtenidos por otros investigadores en el proceso de validación de un método para la cuantificación de Al como adyuvante en vacunas, cuantificado por EAA-F (Mishra et al. 2007).

El resultado para linealidad también es similar al obtenido por otros investigadores, quienes evaluaron cuatro métodos diferentes para la digestión de tejido de ratones albinos Swiss y posterior cuantificación de Al mediante EAA-HG (Bohrer et al. 2003).

En otro trabajo de investigación, Correia et al. 2006 validaron un método analítico para determinar Al, cobre y plomo en uvas mediante EAA-HG (Correia et al. 2006). Sin embargo, no evaluaron la linealidad mediante el coeficiente de correlación, sino que establecieron el rango de linealidad para Al. Éste fue de 1.1 a $50 \mu \mathrm{g} / \mathrm{L}$, a diferencia de nuestro trabajo, en el cual se estableció entre 20 y $100 \mu \mathrm{g} / \mathrm{L}$ de Al.

Exactitudy precisión. En el cuadro II se muestran los resultados obtenidos para exactitud y precisión en 
CUADRO II. ESTIMACIÓN DE LA EXACTITUD Y PRECISIÓN DE UN MATERIAL DE REFERENCIA CERTIFICADO (CRM-S-B) POR ESPECTROSCOPIA DE ABSORCIÓN ATÓMICA POR FLAMA (EAA-F) Y HORNO DE GRAFITO (EAA-HG)

\begin{tabular}{lcc}
\hline Réplica* & $\begin{array}{c}\text { Al }(70 ~ \mathrm{mg} / \mathrm{L}) \\
\text { EAA-F }\end{array}$ & $\begin{array}{c}\mathrm{Al}(70 \mu \mathrm{g} / \mathrm{L}) \\
\text { EAA-HG }\end{array}$ \\
\hline 1 & 70.2 & 69.5 \\
2 & 71.2 & 68.9 \\
3 & 70.4 & 69.1 \\
4 & 70.4 & 68.7 \\
5 & 70.0 & 68.8 \\
6 & 70.4 & 67.8 \\
7 & 70.2 & 67.1 \\
8 & 70.0 & 66.9 \\
9 & 71.2 & 69.2 \\
10 & 70.4 & 68.3 \\
Promedio & 70.4 & 68.4 \\
DS & 0.429 & 0.890 \\
\% Rec & 100.62 & 97.75 \\
\% CV & 0.610 & 1.301 \\
\hline
\end{tabular}

*Cada réplica se realizó por triplicado, $n=30$

DS: desviación estándar, \% Rec: \% de recuperación, \% CV: \% de coeficiente de variación, CRM-S-B: certified reference material-soil-solution B

ambos métodos. Se puede apreciar que la exactitud evaluada mediante el \% Rec fue de 100.62 y 97.75 para EAA por flama y horno de grafito, respectivamente. En tanto que la precisión evaluada mediante el $\%$ CV fue de 0.610 y 1.301 para EAA por flama y horno de grafito, respectivamente.

Los resultados obtenidos se consideran aceptables, ya que el criterio de aceptación para exactitud es de $100 \pm 5 \%$ respecto del porcentaje de recuperación cuando se utiliza un material de referencia certificado (CRM-S-B) y el criterio de aceptación para la precisión es $\% \mathrm{CV} \leq 2$ para métodos instrumentales (ISO 1994, Thompson et al. 2002).

Los resultados obtenidos para exactitud y precisión son similares a los obtenidos por otros investigadores (Mishra et al. 2007) para Al en vacunas (\% Rec de 93.26 a 103.41 y \% CV de 1.62). Estos resultados son similares a los obtenidos durante la validación del método para cuantificar $\mathrm{Cu}$ y otros metales (entre ellos Al) en frutos del olivo (aceituna) mediante EAA-HG, con \% Rec mayores de 95 y coeficientes de variación instrumental de $3 \%$ (Soares et al. 2006).

Repetibilidad. La precisión también se evaluó en condiciones de repetibilidad, realizada en las mismas condiciones de trabajo (el mismo analista, el mismo equipo, tiempos cortos) (Liang et al. 2013). Los resultados obtenidos se muestran en el cuadro III, donde puede apreciarse que el método es repetible para las tres concentraciones de $\mathrm{Al}$ determinado por EAA-F y para las tres concentraciones de $\mathrm{Al}$ determinado mediante EAA-HG, con un nivel de significancia del $5 \%(\alpha=0.05)$ y para $n=25$. Estos resultados de repetibilidad son similares a los obtenidos durante la validación del método para determinar la concentración de Al en uvas (Correia et al. 2006).

CUADRO III. ANÁLISIS DE PRECISIÓN EN CONDICIONES DE REPETIBILIDAD PARA TRES CONCENTRACIONES DIFERENTES DEAL, DETERMINADO POR ESPECTROSCOPIA DE ABSORCIÓN ATÓMICA POR FLAMA (EAA-F) Y HORNO DE GRAFITO (EAA-HG)

\begin{tabular}{rrrl}
\hline Al & F calculada & F tablas & Decisión \\
\hline \multicolumn{4}{c}{ EAA-F } \\
$20 \mathrm{mg} / \mathrm{L}^{*}$ & 1.4144 & 2.8660 & Repetible \\
$50 \mathrm{mg} / \mathrm{L}^{*}$ & 2.3872 & 2.8660 & Repetible \\
$100 \mathrm{mg} / \mathrm{L}^{*}$ & 2.5780 & 2.8660 & Repetible \\
\hline \multicolumn{4}{c}{ EAA-HG } \\
$20 \mu \mathrm{g} / \mathrm{L}^{*}$ & 1.3070 & 2.8660 & Repetible \\
$40 \mu \mathrm{g} / \mathrm{L}^{*}$ & 1.6787 & 2.8660 & Repetible \\
$60 \mu \mathrm{g} / \mathrm{L}^{*}$ & 2.1921 & 2.8660 & Repetible \\
\hline
\end{tabular}

*Cinco replicas por semana durante cinco semanas $(n=25$, $\alpha=5 \%$ )

F: prueba estadística de Fisher

Límite de detección (LD) y límite de cuantificación $(L C)$. Los resultados obtenidos respecto de estas variables para $\mathrm{Al}$ determinado por EAA-F fueron de 0.06 y $0.19 \mathrm{mg} / \mathrm{L}$ de $\mathrm{Al}$ y de 0.05 y $0.18 \mu \mathrm{g} / \mathrm{L}$ de $\mathrm{Al}$ determinado por EAA-HG. Estos resultados son menores que los reportados para $\mathrm{Al}$ como adyuvante en vacunas determinado por EAA-F $(\mathrm{LD}=0.23 \mathrm{mg} / \mathrm{L}$ y LC $=1.11 \mathrm{mg} / \mathrm{L})$ (Mishra et al. 2007), y también menores que los reportados en trabajos previos por Correia et al. (2006) y Soares et al. (2006) (LD = $1.11 \mu \mathrm{g} / \mathrm{L}$ y $\mathrm{LC}=3.70 \mu \mathrm{g} / \mathrm{L}$ de $\mathrm{Al}$ determinado por EAA-HG).

Material de referencia certificado. Ya que no fue posible utilizar un material de referencia certificado de tejido de ratas, el proceso de validación del método se realizó utilizando un material de referencia certificado de tejido de ostión (NIST-1566b) que tiene una concentración de $\mathrm{Al}$ de $197.2 \pm 6 \mathrm{mg}$ de $\mathrm{Al} / \mathrm{kg}$ de tejido. 
CUADRO IV. PORCENTAJES DE RECUPERACIÓN DE MUESTRAS FORTIFICADAS CON $100 \mathrm{mg} / \mathrm{L}$ DE ALUMINIO PARA ESPECTROSCOPIA DE ABSORCIÓN ATÓMICA POR FLAMA (EAA-F), MUESTRAS FORTIFICADAS CON $200 \mu \mathrm{g} / \mathrm{L}$ DE AL PARA ESPECTROSCOPIA DE ABSORCIÓN ATÓMICA POR HORNO DE GRAFITO (EAA-HG) Y MUESTRAS TESTIGO SIN FORTIFICAR, ASÍ COMO PORCENTAJE DE RECUPERACIÓN PARA UN MATERIAL DE REFERENCIA CERTIFICADO $(n=25)$

\begin{tabular}{lccc}
\hline Muestras & $\begin{array}{c}\text { Testigo sin } \\
\text { fortificar }(\mathrm{mg} / \mathrm{L})\end{array}$ & $\begin{array}{c}\text { Muestras fortificadas } \\
(\% \text { Rec) EAA-F }\end{array}$ & $\begin{array}{c}\text { Muestras fortificadas } \\
(\% \text { Rec) EAA-HG }\end{array}$ \\
\hline Mama & 0.31 & 100.91 & 109.00 \\
Hígado & 0.22 & 103.52 & 93.50 \\
Hueso & 0.37 & 100.64 & 105.00 \\
Cerebro & 0.33 & 109.00 & 104.00 \\
Alimento para ratas & 0.47 & 99.32 & 101.21 \\
Agua para ratas & 0.03 & 107.03 & 100.23 \\
Blanco de digestión & 0.05 & 100.44 & 100.12 \\
MRC NIST 1566b* & - & 100.60 & 100.51 \\
\hline
\end{tabular}

*MRC: material de referencia certificado (CRM NIST 1566b)

REC: porcentaje de recuperación, EAA-F: espectroscopia de absorción atómica por flama, EAA-HG: espectroscopia de absorción atómica por horno de grafito

Los resultados obtenidos para la exactitud a través del \% Rec fueron de 100.60 para EAA-F y de 100.51 para EAA-HG (Cuadro IV). El criterio de aceptación para un material de referencia certificado es de $100 \pm 5 \%$ (CENAM-EMA 2012). Estos resultados representan un valor muy cercano al $100 \%$ y constituyen un rango más estrecho respecto a los resultados obtenidos por Correia et al. (2006) al utilizar hojas de espinaca (NIST-1570a) como material de referencia certificado en sustitución de las uvas, cuyo porcentaje de recuperación fue del $97 \%$ para Al.

No obstante, Bohrer et al. (2003) reportan valores de recuperación del 89 al $121 \%(105 \pm 16)$ para Al utilizando hígado de pollo como material de referencia certificado en sustitución de tejido de ratón albino Swiss.

\section{Aplicabilidad del método validado}

El método validado se utilizó en tejidos de rata (mama, hígado, hueso y cerebro), agua de beber y alimento para ratas sin fortificar y fortificado con 100 $\mathrm{mg} / \mathrm{L}$ de estándar de Al. Los tejidos de rata Sprague Dawley, agua de beber y alimento para ratas fueron digeridos en horno de microondas.

Los resultados obtenidos se muestran en el cuadro IV. Como se puede apreciar, los porcentajes de recuperación de $\mathrm{Al}$ en los diferentes tejidos de rata, alimento, agua y controles se encuentran dentro del rango recomendado de $100 \pm 15 \%$ (CENAM-EMA 2012). Es importante mencionar que no se detectó presencia de $\mathrm{Al}$ en los blancos de digestión para EAA-F.

\section{Consideraciones adicionales}

Una vez que se ha validado un método analítico y se ha demostrado su aplicabilidad (González y Herrador 2007), la normatividad internacional establece que es necesario estimar la incertidumbre para darle plena confiabilidad a los resultados obtenidos (ISO 2005). Si bien hay una gran cantidad de artículos científicos relacionados con diversas metodologías analíticas para cuantificar Al en diferentes tipos de tejidos de animales de experimentación o en humanos, la mayoría no realizan o no consideran el proceso de validación del método empleado.

En la literatura científica hay pocos trabajos enfocados a este proceso de validación, y son menos aun los que estiman la incertidumbre después de validar el método analítico (Krouwer 2003, Theodorsson 2012). En este sentido, continuaremos el proceso analítico de esta metodología validada y en trabajos futuros presentaremos los datos relacionados con la estimación de la incertidumbre.

\section{CONCLUSIONES}

Se ha logrado validar un método analítico para cuantificar Al en tejidos de ratas mediante EAA por flama y por horno de grafito, utilizando criterios establecidos por la normatividad internacional vigente. El proceso de validación para este método analítico puede servir de referencia para investigaciones posteriores relacionadas con $\mathrm{Al}$ y sus efectos en animales 
de experimentación. Aunque este método analítico ha sido validado, aún falta la verificación de las autoridades regulatorias, así como revisar, analizar y repetir los ensayos y pruebas para que pueda considerarse un método de referencia. Se necesitan ensayos de validación interlaboratorios, así como la implementación de métodos analíticos que permitan cuantificar $\mathrm{Al}$ en condiciones similares pero utilizando técnicas diferentes como espectroscopia de emisión atómica $\mathrm{y}$ fluorescencia de rayos X, entre otras.

\section{AGRADECIMIENTOS}

Agradecemos a los departamentos de Ciencias Químico-Biológicas y de Ingeniería Química y Metalurgia de la Universidad de Sonora el financiamiento y las facilidades para realizar este proyecto de investigación. También a la Dra. Leticia García Rico, del Centro de Investigación en Alimentación y Desarrollo, por las facilidades otorgadas para realizar la digestión de las muestras en el horno de microondas MARSxpress 5.

\section{REFERENCIAS}

ATSDR (2008). Toxicological profile for aluminum. Agency for Toxic Substances and Disease Registry, Department of Health and Human Services, Division of Toxicology and Environmental Medicine, Atlanta, Georgia, EUA, 257 pp.

Batista E., Almeida N., Filipe E. y Alves J.S. (2009). Comparison of two different approaches in the uncertainty calculation of gravimetric volume calibration. En: Procedures of the XIX IMEKO World Congress Fundamental and Applied Metrology. Lisboa, Portugal, 6 al 11 de septiembre, 2343-2345.

Blair R.C. y Taylor R.A. (2007). Biostatistics for health science. 1a. ed. Pearson-Prentice Hall, Nueva York, 45 pp.

Bohrer D., do Nascimento P.C., Binotto R. y Becker E.J. (2003). Influence of the glass packing on the contamination of pharmaceutical products by aluminum. Part II1: Interaction container-chemicals during the heating for sterilization. J. Trace Elem. Med. Biol. 17 (2), 107-115. DOI: $10.1016 / \mathrm{S} 0946-672 \mathrm{X}(03) 80006-8$

Bohrer D., Dessuy M.B., Kaizer R., Nascimento P.C., Schetinger M.R.C., Morsch V.M., de Carvalho L.M. y Garcia S.C. (2008). Tissue digestion for aluminum determination in experimental animal studies. Anal. Biochem. 377, 120-127.

DOI: $10.1016 /$ j.ab.2008.03.029
CENAM-EMA (2012). Guía técnica de trazabilidad metrológica e incertidumbre de medida en las mediciones analíticas que emplean la técnica de espectrofotometría de absorción atómica con plasma acoplado inductivamente. Guía Técnica de Laboratorio. Centro Nacional de Metrología-Entidad Mexicana de Acreditación, México, 28 pp.

CEM Corporation (2014). MARSxpress 5. Operation manual. Mathews, Carolina del Norte, EUA, 23 pp.

Correia L., Soares M.E. y Bastos M.L. (2006). Validation of an electrothermal atomization atomic absorption spectrometry method for the determination of aluminum, copper, and lead in grapes. J. Agric. Food Chem. 54 (25), 9312-9316. DOI:10.1021/jf0620564

Di Virgilio A.L., Reigosa, M., Arnal, P.M., Fernández, M. y de Mele L. (2010). Comparative study of the cytotoxic and genotoxic effects of titanium oxide and aluminum oxide nanoparticles in Chinese hamster ovary (CHOK1) cells. J. Hazard Mater. 177 (1-3), 711-718. DOI: 10.1016/j.jhazmat.2009.12.089

EMEA (2009). Recommendation on the evaluation of the benefit-risk balance of veterinary medicinal products. Official guide. Committee for Medicinal Products for Veterinary Use (CVMP), European Medicines Agency, Londres, Reino Unido, $56 \mathrm{pp}$.

FDA (2014). CFR-Code of Federal Regulations, title 21, chapter I, subchapter E, part 511. Revised as of April 1. Food and Drug Administration, Silver Spring, Maryland, EUA, 43 pp.

González A.G. y Herrador M.A. (2007). A practical guide to analytical method validation, including measurement uncertainty and accuracy profiles. TrAC 26 (3), 227-238.

DOI:10.1016/j.trac.2007.01.009

ISO (1994). ISO 5725-2. Part 2. Accuracy (trueness and precision) of measurement methods and results-Part 2: Basic method for the determination of repeatability and reproducibility of a standard measurement method. International Organization for Standardization, Ginebra, Suiza, 14 pp.

ISO (2005). ISO/IEC 17025:2005. Requisitos generales para la competencia de los laboratorios de ensayo y de calibración. International Organization for Standardization, Ginebra, Suiza, 23 pp.

Krewsky D., Yokel R.A., Neibor E., Borchelt D., Cohen J., Harry J.S., Kacew S., Lindsay J., Mahfouz A.M. y Rondeau V.J. (2007). Human health risk assessment for aluminum, aluminum oxide, and aluminum hydroxide. J. Toxicol. Environ. Health B Crit. Rev. 10 (1), 1-269.

DOI: $10.1080 / 10937400701597766$

Krouwer J.S. (2003). Point critique of the guide to the expression of uncertainty in measurement method of 
estimating and reporting uncertainty in diagnostic assays clinical. Clin. Chem. 49 (11), 1818-1821.

DOI: 10.1373/clinchem.2003.019505

Liang D., Steinert C., Bammesberger S., Tanguy L., Ernst A., Zengerleand R. y Koltay P. (2013). Novel gravimetric measurement technique for quantitative volume calibration in the submicroliter range. Meas. Sci. Technol. 24 (2), 1-10. DOI:10.1088/0957-0233/24/2/025301

Lorefice S. (2009). Traceability and uncertainty analysis in volume measurements. Measurement 42 (10), 15101515. DOI: 10.1016/j.measurement.2009.07.016

Mahieu S., Contini M.C., González M. y Millen N. (2009). Melatonin reduces oxidative damage induced by aluminum in rat kidney. Toxicol. Lett. 190 (1), 1-9. DOI: $10.1016 /$ j.toxlet.2009.06.852

Mishra A., Bhalla S.R., Rawat S., Vansal B., Sehgal R. y Kumar S. (2007). Standardization and validation of a new atomic absorption spectroscopy technique for determination and quantitation of aluminum adjuvant in immunobiologicals. Biologicals 35, 277-284.

DOI: 10.1016/j.biologicals.2007.03.003

Perkin Elmer (2012). Manual de operación de equipo de espectroscopía de absorción atómica modelo AAnalyst 400. México D.F., México, 98 pp

Prozialeck W.C., Edwards J.R. y Nebert D.W. (2008). The vascular system as a target of metal toxicity. Toxicol. Sci. 102 (2), 207-218. DOI:10.1093/toxsci/kfm263

Soares M.E., Pereira J.A. y Bastos M.L. (2006). Validation of a method to quantify copper and other metals in olive fruit by ETAAS. Application to the residual metal control after olive tree treatments with different copper formulations. J. Agric. Food Chem. 54 (11), 3923-3928.

DOI: $10.1021 / \mathrm{jf0608930}$

Tchounwou P.P., Yedjoum C.G., Patlolla A.K. y Sutton D.J. (2012). Heavy metal toxicity and the environment. EXS 101, 133-164.

DOI: 10.1007/978-3-7643-8340-4_6

Theodorsson E. (2012). Validation and verification of measurement methods in clinical chemistry. Bioanalysis 4 (3), 305-320. DOI: 10.4155/bio.11.311

Thompson M., Ellison S.L.R. y Wood R. (2002). IUPAC technical report. Harmonized guidelines for singlelaboratory validation of methods of analysis. Pure Appl. Chem. 74 (5), 835-855.

DOI: $10.1351 / \mathrm{pac} 200274050835$

Yamamoto S., Tomoda H. y Watanabe S. (2007). Watersoluble metal working fluids additives derived from the esters of acid anhydrides with higher alcohols for aluminum alloy materials. J. Oleo Sci. 56 (9), 463-469. DOI: $10.5650 /$ jos.56.463

Yokel R.A., Hicks C.L. y Florence R.L. (2008). Aluminum bioavailability from basic sodium aluminum phosphate, an approved food additive emulsifying agent, incorporated in cheese. Food Chem. Toxicol. 46 (6), 2261-2266. DOI: 10.1016/j.fct.2008.03.004

Van Loon J.C. (1985). Selected methods of trace metal analysis: Biological and environmental samples. Chemical analysis. A series of monographs on analytical chemistry and its applications. 1a ed. John Wiley and Sons, Nueva York, EUA, 65 pp. 